\title{
REDUÇÃO DO EFEITO ANALGÉSICO DA ESTIMULAÇÃO ELÉTRICA NERVOSA TRANSCUTÂNEA DE BAIXA FREQÜÊNCIA EM RATOS TOLERANTES À MORFINA
}

\author{
Resende MA ${ }^{1}$, Gonçalves HH ${ }^{2}$, Sabino GS ${ }^{2}$, Pereira LSM ${ }^{1}$ e Francischi JN ${ }^{3}$ \\ ${ }^{1}$ Departamento de Fisioterapia, Escola de Educação Física, Fisioterapia e Terapia Ocupacional da Universidade Federal \\ de Minas Gerais -UFMG, Belo Horizonte, MG - Brasil \\ ${ }^{2}$ PPG Ciências da Reabilitação, Departamento de Fisioterapia, UFMG, Belo Horizonte, MG - Brasil \\ ${ }^{3}$ Departamento de Farmacologia do Instituto de Ciências Biológicas, UFMG, Belo Horizonte, MG - Brasil \\ Correspondência para: Marcos Antônio de Resende, Departamento de Fisioterapia, Escola de Educação Física, \\ Fisioterapia e Terapia Ocupacional, UFMG, Av. Antônio Carlos, 6627, São Francisco, CEP 31270-901, Belo Horizonte, \\ MG, - Brasil, e-mail: mresende@eeffto.ufmg.br
}

Recebido: 29/04/2005 - Aceito: 28/03/2006

\begin{abstract}
RESUMO
Objetivo: Investigar o efeito da TENS de baixa $(10 \mathrm{~Hz})$ e alta freqüência $(130 \mathrm{~Hz})$ aplicadas na pata inflamada do rato após tratamento crônico com morfina. Método: Foram utilizados 140 ratos Holtzman fêmeas, nos quais a carragenina $(\mathrm{Cg} 250 \mu \mathrm{g} / 0,1 \mathrm{ml})$ foi administrada na pata posterior direita para a indução da inflamação. TENS de baixa e alta freqüência foi aplicada por 20 min, após 2 h e 30 min da Cg e seu efeito medido através do método de Randall-Selitto. O antagonista opióide Naltrexona (3mg/kg,sc), foi administrado 30 minutos antes da TENS para verificar a liberação de substâncias opióides endógenas. A tolerância foi obtida após administração da morfina $(10 \mathrm{mg} / \mathrm{kg}, \mathrm{sc})$, duas vezes ao dia, durante sete dias. $\mathrm{O}$ tratamento com TENS de baixa e alta freqüência foi realizado no oitavo dia às $2 \mathrm{~h}$ e $30 \mathrm{~min}$ após $\mathrm{Cg}$. A análise estatística foi feita pelo método da análise de variância ANOVA (One Way) seguido de um teste "post hoc" (Teste de Bonferroni), com nível de significância quando p < 0,05. Resultados: TENS de baixa e alta freqüência inibiu em $100 \%$ a hiperalgesia induzida pela $\mathrm{Cg}$. Animais tratados previamente com naltrexona mostraram completa reversão da analgesia induzida pela baixa freqüência mas não pela alta freqüência. Após tolerância à morfina, os valores da TENS de baixa freqüência indicaram total ausência de analgesia, ao contrário da TENS de alta freqüência que induziu anti-hiperalgesia. Conclusão: Conclui-se que a atividade analgésica da TENS de baixa freqüência é reduzida após o desenvolvimento de tolerância a morfina.
\end{abstract}

Palavras-chave: TENS, dor, morfina, tolerância, carragenina, eletroterapia.

\section{ABSTRACT \\ Reduction in Analgesic Effect from Low-Frequency Transcutaneous Electrical Nerve Stimulation In Morphine-Tolerant Rats}

Objective: To investigate the effects of low $(10 \mathrm{~Hz})$ and high-frequency $(130 \mathrm{~Hz})$ transcutaneous electrical nerve stimulation (TENS) applied to inflamed paws of rats following chronic treatment with morphine. Method: 140 female Holtzman rats were utilized. Carrageenan $(250 \mu \mathrm{g} / 0.1 \mathrm{ml})$ was administered to the right hind paws to induce inflammation. Two and a half hours after carrageenan injection, low and high frequency TENS was applied to the inflamed paw for 20 min, and its effect was measured via the Randall-Selitto method. The opioid antagonist naltrexone ( $3.0 \mathrm{mg} / \mathrm{kg}$, subcutaneously) was administered $30 \mathrm{~min}$ before TENS, to verify the release of endogenous opioids. Morphine tolerance ( $10 \mathrm{mg} / \mathrm{kg}$, subcutaneously) was induced by twice-daily injection over seven days. Low and high frequency TENS treatment was carried out on the eighth day, 2.5 hours after carrageenan injection. Statistical analysis was performed using one-way analysis of variance (ANOVA), followed by the post hoc Bonferroni test, with a significance level of $\mathrm{p}<0.05$. Results: Both low and high frequency produced $100 \%$ inhibition of carrageenan-induced hyperalgesia. Naltrexone-treated animals showed complete reversion of analgesia induced by low but not high-frequency TENS. After attaining morphine tolerance, the low-frequency TENS values indicated complete absence of analgesia, whereas highfrequency TENS induced anti-hyperalgesia. Conclusion: The analgesic activity of low-frequency TENS is reduced following the development of morphine tolerance.

Key words: TENS, pain, morphine, tolerance, carrageenan, electrotherapy. 


\section{INTRODUÇÃO}

Estimulação elétrica nervosa transcutânea (TENS) é um método não invasivo utilizado para reduzir a dor, mas não o edema de condições inflamatórias ${ }^{1}$. O mecanismo de ação analgésica da TENS não está bem estabelecido. A TENS pode ser aplicada no local da hiperalgesia, uma resposta aumentada ao estímulo nocivo, ou próximo a ela, e os parâmetros de aplicação como intensidade, freqüência e duração do pulso quando manipulados podem afetar a eficiência da TENS $^{2,3}$. Em estudos com animais, TENS de baixa $(<10 \mathrm{~Hz})$ e alta freqüência $(>100 \mathrm{~Hz})$ têm reduzido a hiperalgesia induzida pela carragenina e kaolin ${ }^{4,5}$, duas substâncias bastante utilizadas no estudo da dor inflamatória ${ }^{3,6}$. Alguns autores têm demonstrado que a TENS de baixa freqüência $(4 \mathrm{~Hz})$ produz analgesia através da ativação de receptores opióides $\mu$, situados na medula espinhal e no bulbo ventral rostral, e a TENS de alta freqüência $(100 \mathrm{~Hz})$ por ativação dos receptores $\delta$ na medula e regiões do bulbo ${ }^{7,8,9,10}$. Em humanos e em animais, foi demonstrado que o efeito analgésico produzido pela TENS de baixa freqüência foi revertido pela naloxona e naltrexona, dois antagonistas opióides, o que não ocorreu com a TENS de alta freqüência ${ }^{1,11}$.

Uma outra forma de reduzir a dor é através da administração de opióide exógeno como a morfina. Sua ação analgésica ocorre como conseqüência de sua interação com receptores específicos $\mu, \delta$, e $\kappa$, localizados em diversos pontos do sistema aferente e eferente, que participam da transmissão da sensibilidade dolorosa e modulam a informação nociceptiva $^{12,13}$. Repetidas administrações de morfina reduz gradualmente o seu efeito analgésico, desencadeando um fenômeno conhecido como tolerância ${ }^{14,16}$. A tolerância à morfina parece estar mais ligada ao receptor $\mu$ do que a outros tipos de receptores opióides ${ }^{18,19}$. Dessa forma, a morfina e a TENS de baixa freqüência, parecem apresentar o mesmo mecanismo de ação.

Poucos estudos na literatura associam a TENS de baixa e alta freqüência com morfina crônica para avaliar a efetividade analgésica da TENS. O objetivo do presente estudo foi verificar em ratos o efeito da TENS de baixa $(10 \mathrm{~Hz})$ e alta freqüência $(130 \mathrm{~Hz})$ aplicadas na pata inflamada do rato após tratamento crônico com morfina.

\section{MATERIAIS E MÉTODOS}

Este estudo foi aprovado pelo Comitê de Ética Animal da Universidade Federal de Minas Gerais (CETEA/UFMG $\left.-\mathrm{n}^{\mathrm{o}} 067 / 03\right)$.

\section{Animais}

Foram utilizados no estudo um total de 140 ratos Holtzman fêmeas, pesando de 160 a 180 gramas fornecidos pelo Centro de Bioterismo do ICB-UFMG. Os animais em número de 4 a 6 por caixa permaneceram na sala de expe- rimentação por dois a três dias antes da realização dos experimentos para adaptação ao aparelho algesimétrico, com livre acesso a água e alimentação, ciclo claro-escuro de 12 h e temperatura controlada $\left(23-25^{\circ} \mathrm{C}\right)$.

\section{Indução de hiperalgesia pela carragenina}

Carragenina $(250 \mu \mathrm{g} / 0,1 \mathrm{ml})$, um polissacarídeo sulfatado que produz reação inflamatória aguda, foi injetada subcutâneamente na superfície plantar posterior direita do rato no tempo zero. A pata contralateral foi injetada com o mesmo volume de salina (veículo).

\section{Medida de hiperalgesia}

A medida de hiperalgesia foi realizada por um experimentador através de um algesímetro da Ugo Basile ${ }^{\circledR}$, que consistiu no deslocamento de um peso de no máximo $500 \mathrm{~g}$ sobre a superfície plantar posterior direita e registrado o limiar de retirada da pata ao estímulo nociceptivo ${ }^{20}$.

\section{Estimulação elétrica nervosa transcutânea}

A estimulação elétrica nervosa transcutânea foi realizada através de um aparelho Neurodyn III (IBRAMED). O aparelho foi previamente calibrado para liberar os seguintes parâmetros: estimulação de baixa $(10 \mathrm{~Hz})$ e alta $(130 \mathrm{~Hz})$ freqüência com duração de pulso de $130 \mu \mathrm{s}$. A intensidade sensorial foi considerada imediatamente abaixo do limiar motor. Um par de eletrodos foi especialmente construído para a estimulação elétrica com $1 \mathrm{~cm}^{2}$ de tamanho, fixados na região dorsal e superfície plantar da pata do rato. Os animais ficavam movimentando livremente na caixa durante a estimulação elétrica.

\section{Tratamento com drogas}

Morfina $(10,0 \mathrm{mg} / \mathrm{Kg}, \mathrm{sc})$, foi utilizada de forma crônica para o desenvolvimento de tolerância ao opióide, e o antagonista opióide naltrexona $(3 \mathrm{mg} / \mathrm{Kg}$, sc), foi administrado para verificar a liberação de substâncias opióides endógenas durante o tratamento com TENS.

\section{Delineamento Experimental}

Para o estudo da atividade pró-inflamatória da carragenina, foram formados três grupos de animais que receberam na pata posterior direita injeção de carragenina $(150,250$ e $500 \mu \mathrm{g} / \mathrm{pata})$ e seu grupo controle recebeu salina. A dose escolhida para os próximos experimentos foi de 250 $\mu \mathrm{g} / \mathrm{pata}$, dada a intensidade intermediária da resposta. É bem conhecida a cinética da carragenina, em que o pico da hiperalgesia ocorre na $3^{\mathrm{a}}$ hora após a sua administração. Por isso, foi feita a medida da hiperalgesia antes (tempo zero) e 1, 2, 3, 4 e $6 \mathrm{~h}$ após a injeção intraplantar de carragenina. Após 2 h e 30 minutos da administração da carragenina, dois grupos de animais foram tratados com TENS de baixa e alta freqüência durante 20 minutos. Os animais controles receberam 
carragenina mas não a TENS para fins de comparação.Em seguida, dois grupos de animais receberam a NALTREXONA por via sistêmica, 30 minutos antes da TENS de baixa e alta freqüência para o estudo da ação analgésica da TENS. Um grupo de animais controle recebeu salina e TENS de baixa freqüência e o outro grupo controle recebeu salina e TENS de alta freqüência. Para o tratamento crônico com morfina, foram formados quatro grupos de animais que receberam duas administrações diárias, com no mínimo 8 horas de intervalo entre uma aplicação e outra, durante 7 dias, conforme protocolo experimental21 ${ }^{21}$ Quatro grupos de animais controle, receberam durante sete dias o mesmo volume de salina por via subcutânea para fins de comparação. No $8^{\circ}$ dia, após o desenvolvimento da tolerância à morfina, os animais foram tratados com TENS de baixa freqüência, alta freqüência e morfina aguda, e os animais controle receberam salina aguda, salina crônica e morfina aguda às 2 h e 30 minutos após a administração da carragenina.

\section{Análise Estatística}

Os animais foram randomicamente distribuídos entre os grupos experimentais e controles. Os resultados obtidos foram analisados pelo método da análise de variância ANOVA (One Way). Para evidenciar entre quais variáveis ocorria a diferença significativa, foi realizado um teste "post hoc" (Teste de Bonferroni), com nível de significância quando $\mathrm{p}<0,05$.

\section{RESULTADOS}

Hiperalgesia foi desenvolvida na pata do rato após administração intraplantar de diferentes doses de carragenina (150, 250 e $500 \mu \mathrm{g} /$ pata), conforme mostrado na fig. 1 . Carragenina comparada com os animais controles (salina), provocou uma hiperalgesia dose -dependente e alcançou um nível máximo na $3^{\mathrm{a}} \mathrm{h}$ após a sua administração, permanecendo até a $6^{\mathrm{a}} \mathrm{h}$.

Conforme mostrado na figura 2, tanto a baixa como a alta freqüência da TENS reverteu completamente a hiperalgesia induzida pela carragenina na $3^{\mathrm{a}} \mathrm{h}$, indicando um efeito analgésico após tratamento com TENS. TENS de baixa freqüência quando comparado com a de alta freqüência causou um efeito analgésico mais duradouro, que foi detectado até a $6^{\mathrm{a}} \mathrm{h}$ após a injeção da carragenina.

Naltrexona foi administrado $30 \mathrm{~min}$ antes do tratamento com TENS de baixa e alta freqüência. $\mathrm{O}$ efeito analgésico da TENS de baixa freqüência foi totalmente revertido da $3^{\mathrm{a}}$ $h$ até a $6^{\mathrm{a}} \mathrm{h}$ após injeção da carragenina, conforme verificado na figura 3. Entretanto, a mesma dose de naltrexona não modificou a analgesia provocada pela TENS de alta freqüência (figura 4).

Após o desenvolvimento de tolerância à morfina, os animais foram tratados com TENS de baixa e alta freqüência. Conforme pode-se observar na fig. 5, os valores obtidos com

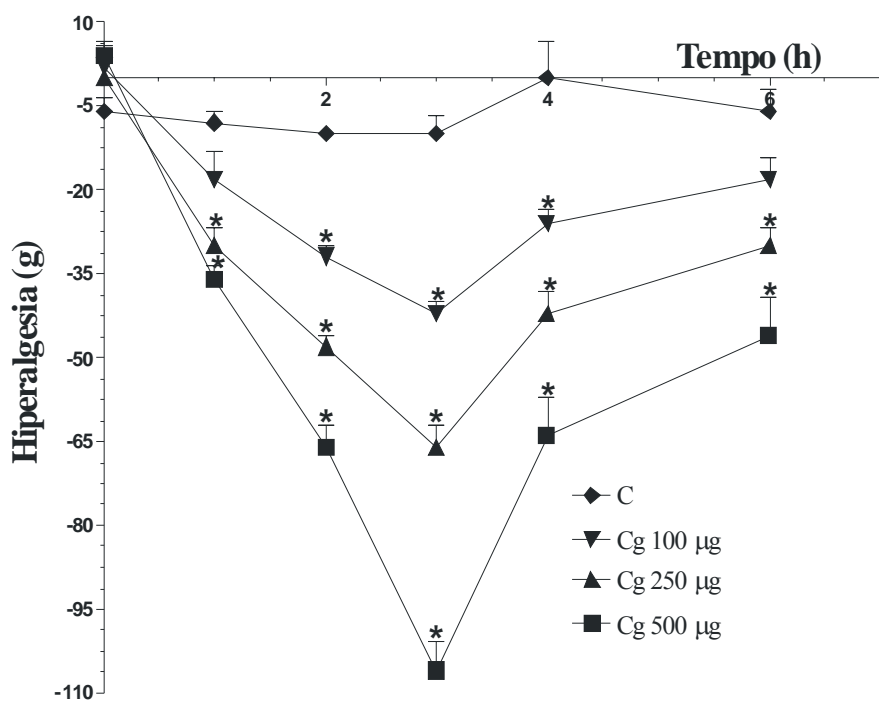

Figura 1. Curva de desenvolvimento da hiperalgesia após administração da carragenina $(\mathrm{Cg})$ na pata do rato. Os resultados estão representados como a diferença entre a pata teste e controle. $\mathrm{N}=5$ animais/grupo. * indica diferença estatisticamente significativa em relação a C.

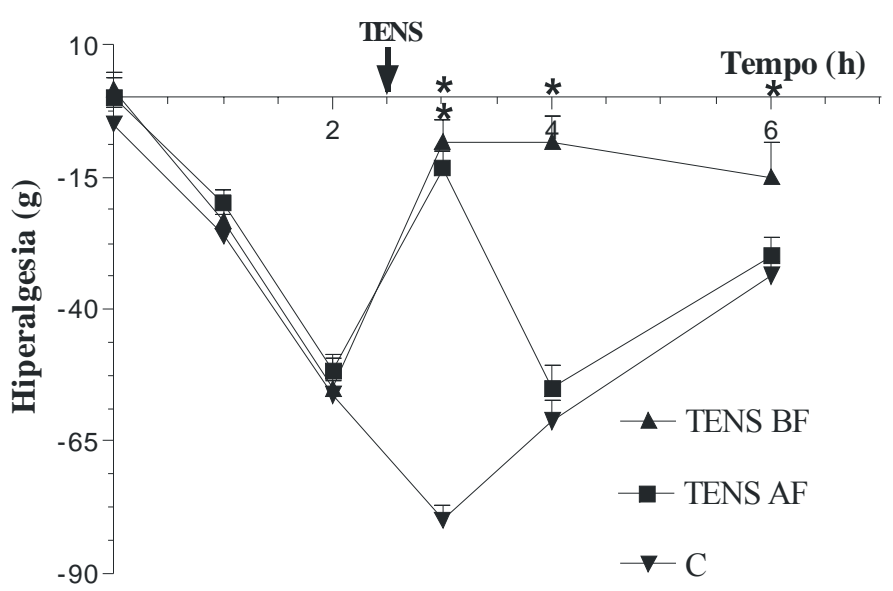

Figura 2. Efeito da baixa (BF) e alta (AF) freqüência da TENS sobre a hiperalgesia da carragenina. $\mathrm{N}=6$ a 8 animais/grupo. * indica diferença estatisticamente significativa em relação a C.

a TENS de baixa freqüência são semelhantes aos do grupo controle, indicando uma total ausência de atividade analgésica. Ao contrário do que se observa com a TENS de alta freqüência (figura 6), mesmo após os animais tornarem-se tolerantes à morfina continuou induzindo efeito analgésico.

\section{DISCUSSÃO}

$\mathrm{O}$ estudo demonstrou que a TENS de baixa freqüência foi menos efetivo que o TENS de alta freqüência em animais tolerantes a morfina. Esses resultados estão de acordo com 


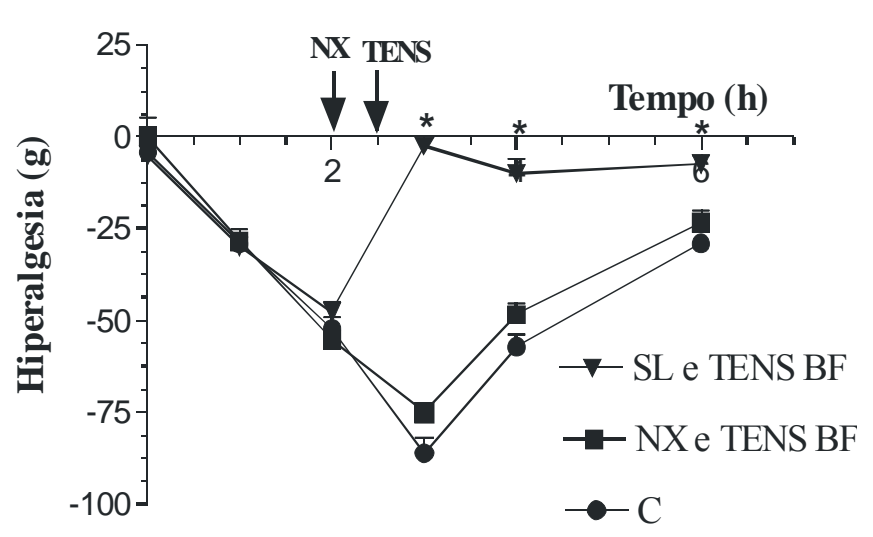

Figura 3. Efeito da naltrexona (NX) sobre a analgesia induzida pela baixa freqüência (BF) da TENS. SL - salina. $\mathrm{N}=4$ a 10 animais/grupo. * indica diferença estatisticamente significativa.

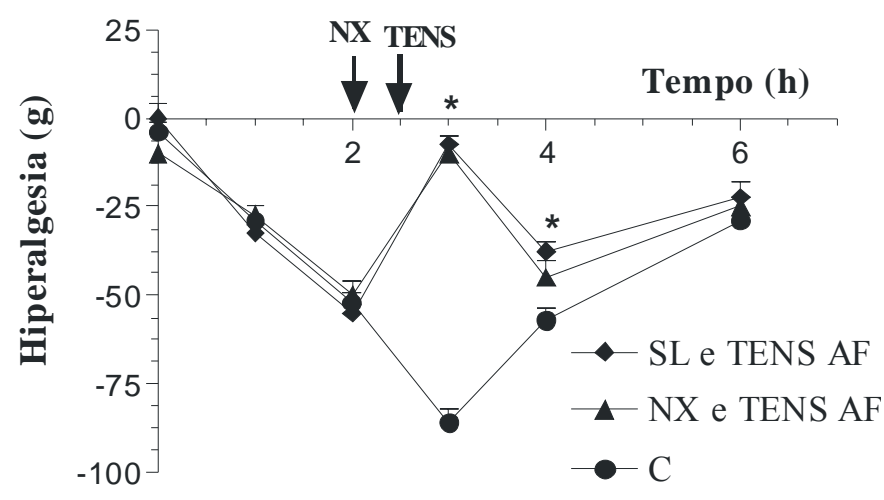

Figura 4. Efeito da naltrexona (NX) sobre a analgesia induzida pela alta freqüência (AF) da TENS. SL - salina. $\mathrm{N}=4$ a 10 animais/grupo. * indica diferença estatisticamente significativa.

outros estudos ${ }^{3}$, que após induzir tolerância à morfina em ratos, verificaram que a TENS de baixa freqüência apresentou uma atividade analgésica bastante reduzida quando comparado ao grupo que recebeu placebo durante o tratamento crônico. Outros autores, também têm demonstrado que repetidas aplicações de TENS de baixa e alta freqüência leva ao desenvolvimento de tolerância a substâncias opióides, com uma correspondente tolerância cruzada à administração de agonistas de receptores opióides $\mu$ e $\delta$, respectivamente ${ }^{22}$. Esses resultados demonstram claramente que o efeito analgésico da TENS de baixa e alta freqüência é mediado por receptor opióide dentro do sistema nervoso central.

Em nosso estudo, TENS de baixa e alta freqüência inibiram em $100 \%$ a hiperalgesia da carragenina. Esses resultados confirmam trabalhos anteriores que obtiveram uma

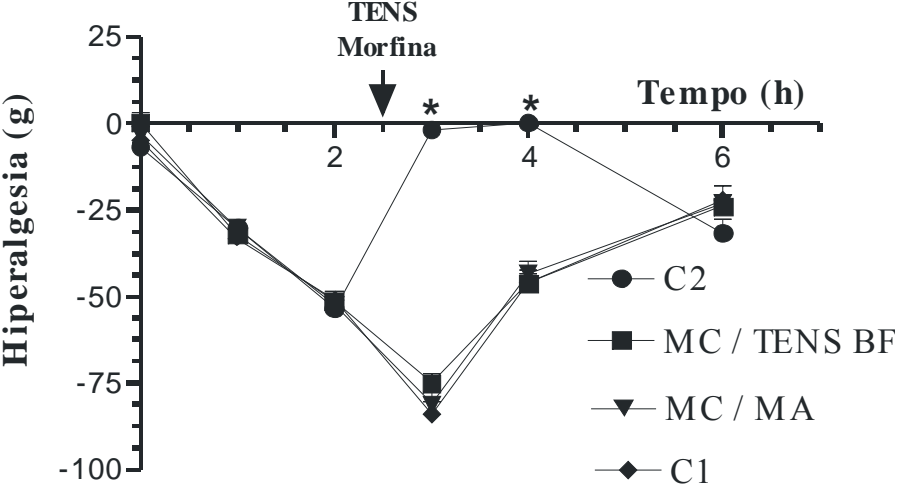

Figura 5. Efeito da TENS de baixa freqüência (BF) em ratos tolerantes à morfina $(\mathrm{MC})$. Morfina aguda (MA). $\mathrm{C} 1$ e $\mathrm{C} 2$ - controles, receberam salina crônica. $\mathrm{C} 1$ recebeu salina aguda e $\mathrm{C} 2$ recebeu $\mathrm{MA}$ às $2 \mathrm{~h}$ e $30 \mathrm{~min}$ após $\mathrm{Cg}$ no $8^{\circ}$ dia. $\mathrm{N}=6$ a 10 animais/grupo. * indica diferença estatisticamente significativa em relação a $\mathrm{C} 2$.

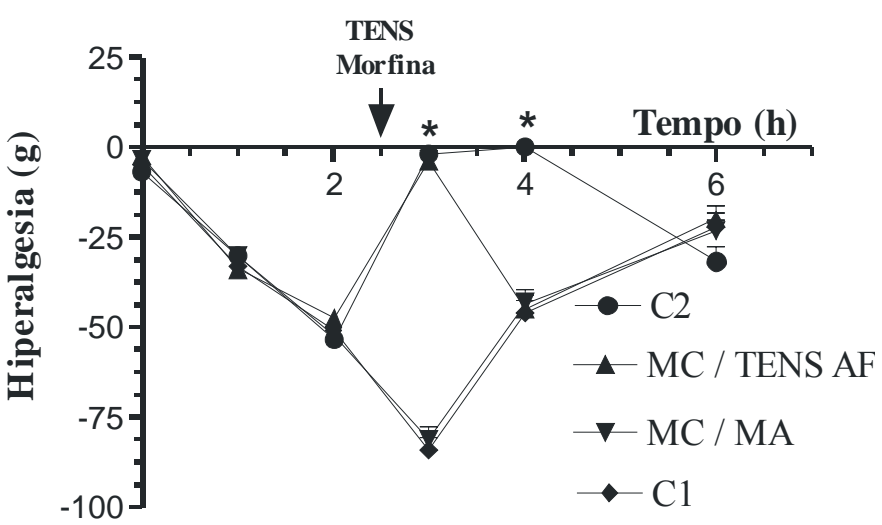

Figura 6. Efeito da TENS de alta freqüência $(\mathrm{AF})$ em ratos tolerantes à morfina (MC). Morfina aguda (MA). C1 e C2 - controles, receberam salina crônica. $\mathrm{C} 1$ recebeu salina aguda e $\mathrm{C} 2$ recebeu $\mathrm{MA}$ às $2 \mathrm{~h}$ e $30 \mathrm{~min}$ após $\mathrm{Cg}$ no $8^{\circ}$ dia. $\mathrm{N}=6$ a 10 animais/grupo. * indica diferença estatisticamente significativa em relação a C2.

redução próxima de $100 \%$ da hiperalgesia secundária induzida pela carragenina e kaolin ${ }^{3}$. Entretanto, devido à sensibilidade do método utilizado neste estudo, não foi possível verificar se a TENS de baixa freqüência quando associada a morfina aguda potencia o seu efeito analgésico. Morfina na dose de $3 \mathrm{mg} / \mathrm{Kg}$ reduziu a hiperalgesia da carragenina quando foi avaliada a resposta ao estímulo mecânico, mas uma dose bem menor de $1 \mathrm{mg} / \mathrm{Kg}$ foi suficiente para reduzir a hiperalgesia quando medida a resposta ao estímulo térmico ${ }^{23}$. Dessa forma, é possível que o método de avaliação mecânica utilizado em nosso estudo, não apresenta a mesma sensibilidade que o método que avalia a resposta ao estímulo térmico ${ }^{24}$. 
Alguns autores propõem que a estimulação elétrica liberada por TENS de baixa freqüência ativa receptores opióides do tipo $\mu$, e libera substâncias analgésicas endógenas ${ }^{3,11}$ promovendo dessa forma uma analgesia mais prolongada. Por outro lado, TENS de alta freqüência deve agir por mecanismos diferentes, através da ativação de receptores opióides $\delta$ na medula espinhal e na região do bulbo ventral rostral ${ }^{10}$. É provável que esta modalidade de TENS ativa fibras nervosas de grande diâmetro na periferia que se projetam para o corno dorsal da medula, e são capazes de ativar o mecanismo do portão espinhal e bloquear o impulso de dor ascendente $^{22}$. Por outro lado, o efeito analgésico produzido pela estimulação elétrica de baixa freqüência é revertido pelo naloxona, um antagonista opióide padrão, mas não o efeito analgésico induzido pela estimulação elétrica de alta freqüência ${ }^{4}$. Através do uso de antagonistas opióides, alguns autores têm estabelecido em modelos animal, que a morfina pode induzir analgesia por ativação de receptores $\mu_{2}$ localizados na região espinhal ou pela ativação de receptores $\mu_{1}$ encontrados em regiões supra-espinhal. Entretanto, quando a morfina é administrada sistemicamente ela atua predominantemente sobre os receptores $\mu_{1}^{19}$. Dessa forma, a TENS de baixa freqüência deve atuar liberando substâncias opióides dentro do sistema nervoso central.

A exposição constante do receptor $\mu$ através da administração diária de morfina em animais pode desenvolver tolerância ao efeito analgésico da morfina ${ }^{16} \mathrm{e}$, consequentemente ao TENS de baixa freqüência, por atuar sobre o mesmo receptor e liberar substâncias opióides endógenas. Dessa forma, conforme podemos observar, nossos resultados confirmam o desenvolvimento de tolerância cruzada entre morfina e TENS de baixa freqüência, mas não a TENS de alta freqüência, que mesmo em animais tolerantes à morfina, continua induzindo analgesia por apresentar mecanismo diferente ao da morfina.

Os resultados apresentados neste trabalho são importantes para a clínica fisioterapêutica, uma vez que a TENS é utilizada para controlar a dor aguda e crônica em diferentes situações. Vários pacientes com doenças oncológicas ou artrite reumatóide em uso prolongado de drogas opióides, são submetidos ao tratamento com TENS. Nestas condições, os parâmetros de aplicação da TENS devem ser observados, uma vez que a TENS de baixa freqüência pode ser menos efetiva que a TENS de alta freqüência. Outros estudos devem ser realizados para a comprovação dos nossos resultados em seres humanos.

\section{REFERÊNCIAS BIBLIOGRÁFICAS}

1. Resende MA, Sabino GG, Cândido CRM, Pereira LSM, Francischi JN. Local transcutaneous electrical stimulation (TENS) effects in experimental inflammatory edema and pain. European Journal of Pharmacology 2004; 504: 217-22.
2. Robinson AJ. Transcutaneous electrical nerve stimulation for the control of pain in musculoskeletal disorders. JOSPT 1996; 24(4): 208-26.

3. Sluka KA, Judge MA, McColley MM, Reveiz PM, Taylor BM. Low frequency TENS is less effective than high frequency TENS at reducing inflammation induced hyperalgesia in morphine-tolerant rats. Eur J Pain 2000; 4: 185-93.

4. Sluka KA, Bailey K, Bogush J, Olson R, Ricketts A. Treatment with either high or low frequency TENS reduces the secondary hyperalgesia observed after injection of kaolin and carrageenan into knee joint. Pain 1998; 77: 97-102.

5. Sluka KA, Deacon M, Stibal A, Strissel S, Terpstra A. Spinal Blockade of opioid receptors prevents the analgesia produced by TENS in arthritic rats. J Pharmacol Exp Ther 1999; 289(2): 840-6.

6. Resende MA, Reis WGP, Pereira LSM, Ferreira W, Garcia MHLP, Santoro MM, et al. Hyperalgesia and edema responses induced by rat peripheral blood mononuclear cells incubated with carrageenin. Inflammation 2001; 25(5): 277-85.

7. Kalra A, Urban MO, Sluka KA. Blockade of opioid receptors in rostral ventral medulla prevents antihyperalgesia produced by transcutaneous electrical nerve stimulation (TENS). J Pharmacol Exp Ther 2001; 298(1): 257-63.

8. Sjolund BH, Eriksson BE. The influence of naloxone on analgesia produced by peripheral conditioning stimulation. Brain Res 1979; 137: 295-301.

9. Fields HL, Basbaum AI. Central nervous system mechanisms of pain modulation. In: Wall PD, Melzack R, editors. Textbook of Pain. $3^{\text {rd }}$ ed. New York: Churchill Livingstone; 1994.

10. Stamford JA. Descending control of pain. Br J Anaesth 1995; 75(2):217-27.

11. Gellert VF, Holtzman SG. Development and maintenance of morphine tolerance and dependence in the rat by scheduled access to morphine drinking solutions. J Pharmacol Exp Ther 1978; 205(3): 536-46.

12. Wong CS, Hsu MM, Chou R, Chou YY, Tung CS. Intrathecal cyclooxygenase inhibitor administration attenuates morphine antinociceptive tolerance in rats. British Journal of Anaesthesia 2000; 85(5): 747-51.

13. Picker MJ, Craft RM, Negus SS, Powell KR, Mattox SR, Jones $\mathrm{SR}$, et al. Intermediate efficacy mu opioids: examination of their morphine-like stimulus effects and response rate-decreasing effects in morphine-tolerant rats. J Pharmacol Exp Ther. 1992; 263 (2): 668-81.

14. Reisine T, Pasternack G. Opioid analgesics and antagonists. In: Goodman A, Gilman HJG, Limbird LE, Molinoff PB, Raymond WR, editors. The Pharmacological Basis of Therapeutics. $9^{\text {th }}$ ed. New York: The McGraw-Hill; 1996.

15. Randall LD, Selitto JJ. A method for measurement of analgesic activity on inflammed tissues. Arch Int Pharmacodyn 1957, 113: 233-49.

16. Resende MA. Efeito da possível participação de íons cálcio na tolerância ao efeito analgésico da morfina em ratos [dissertação de mestrado]. Belo Horizonte: Universidade Federal de Minas Gerais; 1988. 
17. Chandran P, Sluka KA. Development of opioid tolerance with repeated TENS administration. Pain 2003; 102: 195-201.

18. Sluka KA. Systemic morphine in combination with TENS produces an increased antihyperalgesia in rats with acute inflammation. J Pain 2000; 1(3): 204-11.

19. Hargreaves K, Dubner R, Brown F, Flores C, Joris J. A new and sensitive method for measuring thermal nociception in cutaneous hyperalgesia. Pain 1988; 32: 77-88.

20. Woolf CJ, Barrett GD, Mitchell D, Myers RA. Naloxone-reversible peripheral electroanalgesia in intact and spinal rats. Eur J Pharmacol 1977; 45:311-14.
21. Melzack R. Prolonged relief of pain by brief, intense transcutaneous somatic stimulation. Pain 1975; 1: 357-73.

22. Garrison DW, Foreman RD. Decreased activity of spontaneous and noxiously evoked dorsal horn cells during transcutaneous electrical nerve stimulation (TENS). Pain 1994; 58: 309-15.

23. Melzack R, Wall PD. Pain mechanisms: a new theory. Science 1965; 150: 971-9.

24. Woolf CJ, Thompson JW. Stimulation fibre-induced analgesia: transcutaneous electrical nerve stimulation (TENS) and vibration. In: Wall PD, Melzack R, editors. Textbook of Pain. $3^{\text {rd }}$ ed. New York: Churchill Livingstone; 1994. 